\title{
Internal dosimetry for alpha emitters radiopharmaceuticals in biological tissue studied with the FLUKA code
}

\author{
Mauro Valente ${ }^{* \dagger}$ \\ Institute of Physics E. Gaviola - CONICET, Córdoba; Argentina \\ LIIFAMIR $^{X}$ - Laboratorio de Investigación e Instrumentación en Física Aplicada a la Medicina \\ e Imágenes por Rayos X - University of Córdoba; Argentina \\ E-mail: valentedfamaf.unc.edu.ar
}

\section{Francisco Malano}

Institute of Physics E. Gaviola - CONICET, Córdoba; Argentina

LIIFAMIR ${ }^{X}$ - Laboratorio de Investigación e Instrumentación en Física Aplicada a la Medicina

e Imágenes por Rayos X - University of Córdoba; Argentina

E-mail: malanolfamaf .unc.edu.ar

\section{Pedro Pérez}

Institute of Physics E. Gaviola - CONICET, Córdoba; Argentina

LIIFAMIR $^{X}$ - Laboratorio de Investigación e Instrumentación en Física Aplicada a la Medicina e Imágenes por Rayos X - University of Córdoba; Argentina

E-mail: pperezlefamaf.unc.edu.ar

\begin{abstract}
Nuclear medicine clinical practices for neoplasic disease diagnose and treatment are based on the incorporation of $\alpha, \beta$ and $\gamma$ radiotracers and radiopharmaceuticals, which might be associated with potential damage. Thus, being necessary accurate dosimetry strategies. In vivo absorbed dose appears as an ideal solution. However, its implementation in clinics does not attain enough reliability. In this sense, different approaches were proposed for internal dosimetry calculations. This work presents a novel analytical-numerical approach for internal dosimetry purposes. Dedicated Monte Carlo simulations were performed by subroutines adapted from the FLUKA code. In-water EDK were evaluated at different photon energies and some typical $\gamma$-emitters radiopharmaceuticals; whereas DPK were obtained for both $\alpha$ - and $\beta$ - emitters. Additionally, EDK and DPK were calculated for several biological tissues.
\end{abstract}

X Latin American Symposium on Nuclear Physics and Applications (X LASNPA),

1-6 December 2013

Montevideo, Uruguay

\footnotetext{
${ }^{*}$ Speaker.

${ }^{\dagger}$ www.famaf.unc.edu.ar/ valente
} 


\section{INTRODUCTION}

Medical practices like nuclear medicine involve radiopharmaceuticals that are employed for therapeutic/diagnostic purposes, thus becoming necessary to minimize potential damage or side effects to nearby structures. Thereby, radioisotopes with short range are preferred [1].

With the incorporation of radionuclides in patient for diagnose or therapy, internal dosimetry framework attempts to calculate the corresponding radiation dose and radiobiological quantities for estimating therapy effectiveness and potential risks. The availability of different proposed strategies for internal dosimetry makes possible to deal dose calculations with different methods.

During the last decades some models were proposed for dosimetry dedicated to therapies with radiopharmaceuticals labeled with radioactive emitters. However, these models are mainly devoted to $\beta$ and $\gamma$ emitters, being $\alpha$ particle treatment modality less investigated and actually not frequently used in clinics [1] [2].

There are different modalities for employing radiopharmaceuticals labeled with $\alpha$ emitters in clinics. Targeted $\alpha$ therapy (TAT) constitutes a selective as well as systemic radiotherapy to primary and metastatic tumors. This technique methodology attains good performance even at a low dose rate and hypoxia regions, thus constituting a valuable treatment option. In fact, monoclonal antibodies that recognize tumor-associated antigens are conjugated to potent $\alpha$ emitting radionuclides to form the alpha-immunoconjugate [2] [3].

From a physical point of view it has to be poined out that $\alpha$ particle with energy around 4 to $9 \mathrm{MeV}$ can deposit about $100 \mathrm{keV} / \mu \mathrm{m}$ within a few cell diameters $(40-90 \mu \mathrm{m})$. Therefore causing direct DNA double-strand breaks, which is considered that lead to cancer cell apoptosis [2]. As known, cell survival is relatively insensitive to the cell cycle or oxygen status for high ionization radiations. Among other relevant advantage, TAT is potent enough to eradicate disseminated cancer cells eliminating also cancer stem cells.

\section{MATERIALS AND METHODS}

Monte Carlo techniques for radiation transport are able to accurately compute absorbed dose in non homogeneous media accounting for different tissues. Simulation of radiation transport across any medium is possible using several Monte Carlo codes and provides accurate dose estimation, achieving voxel-level dose mapping.

An integrate internal dosimetry system requires dedicated developments, the inclusion of different methods regarding basic physics to justify the proposed models.

\subsection{Developed theoretical background}

Typical quantities in internal dosimetry, like specific absorbed dose fraction, photon energy deposition kernel $(E D K)$ and charged particle dose point kernels $(D P K)$ are obtained from Boltzmann radiation transport main equation, according to expression 2.1 that applies to time independent radiance $\mathbf{R}$ (steady state radiation transport equation).

$$
\vec{\Omega} \cdot \vec{\nabla}(R(\vec{r}, \vec{\Omega}))+\sigma_{T}(\vec{r}) R(\vec{r}, \vec{\Omega})=\sigma_{S}(\vec{r})\left[\int_{\Omega^{\prime}} R(\vec{r}, \vec{\Omega}) p\left(\overrightarrow{\Omega^{\prime}}, \vec{\Omega}\right) d \Omega^{\prime}+S(\vec{r}, \vec{\Omega})\right]
$$


Parameters refer to extinction $\left(\sigma_{T}\right)$, scattering $\left(\sigma_{S}\right)$ and absorption $\left(\sigma_{A} \equiv \sigma_{T}-\sigma_{S}\right)$ tensors, $p\left(\vec{\Omega}^{\prime}, \vec{\Omega}\right)$ is the phase function and $S(\vec{r}, \vec{\Omega})$ represents the source term.

The proposed approach models $\mathbf{R}$ angular components in terms of the spherical harmonics base $\left(Y_{i, j}(\hat{\Omega})\right)$ keeping the first more relevant contributions $\left(c_{i, j}\right)$.

$$
\begin{aligned}
R(\vec{r}, \vec{\Omega}) & =\sum_{i=0}^{\infty} \sum_{j=-i}^{i} c_{i, j}(\vec{r}) Y_{i, j}(\hat{\Omega}) \approx \sum_{i=0}^{1} \sum_{j=-i}^{i} c_{i, j}(\vec{r}) Y_{i, j}(\hat{\Omega}) \\
& =c_{0,0} Y_{0,0}+\sum_{j=-1}^{1} c_{i, j}(\vec{r}) Y_{i, j}(\hat{\Omega})=\frac{\Psi(\vec{r})+3 \vec{j}(\vec{r})}{4 \pi}
\end{aligned}
$$

where $\Psi$ is the scalar fluence and $\mathrm{t} \vec{j}$ vector irradiance or current density.

Considering the simplest case of point source in homogeneous isotropic medium: $\sigma_{T}, \sigma_{S}$ and $\sigma_{S}$ tensors become coefficients thus the scattering component $R_{S}$ can be calculated from the primary $R_{P}$ (scattering production from source). Hence,

$$
R_{S}(r, \theta)=-\sigma_{T} R_{S}(r, \theta)+2 \pi\left|r-r_{0}\right|^{2} \sigma_{S} \int_{0}^{\pi} R_{S}(r, \theta) P(\theta) \sin (\theta) d \theta+\sigma_{S} Q(r, \theta)
$$

Thus, the problem is reduced to three relevant phase state variables (energy $E$, radial distance to the source $r$ and angle $\theta$ between $\hat{s}$ and $\hat{\ell}$ ) and $Q(r, \theta)$ is calculated in reference to $R_{P}$ by means of the primary flux $\Phi_{P}$.

Arbitrary source can be described by discretization of time dependent source as sum of time pulses emitting spectrum of $N$ energy channels at time $t_{k}\left(R_{P}^{k}(r, t)\right)$.

$$
Q(r, t)=4 \pi \Phi_{P}\left(r_{0}\right) e^{-\sigma_{T}\left|r-r_{0}\right|} \sum_{k \geq 0} q_{k} \delta\left(t_{k}-t_{0}\right) \quad R_{P}^{k}(r, E, t) \approx \sum_{n=1}^{N} \frac{\Phi_{P}^{n}\left(r_{0}\right)}{s^{2}} e^{-\sigma_{T}\left(E_{n}\right) s} q_{k}
$$

where $q_{k}$ is the total source emission intensity corresponding to the pulse at $t_{k}$.

\subsection{Implementation of analytical model for voxel-level internal dosimetry}

Scaled DPK are obtained from the above expressions integrating over all active source points. Abdsorbed dose $D(\vec{r})$ is assessed by the convolution of kernel $\mathbf{K}(\vec{r})$ and cumulative activity $\mathbf{A}(\vec{r})$ tensors.

$$
D(\vec{r})=\mathbf{K}(\vec{r}) \otimes \mathbf{A}(\vec{r})=\int_{-\infty}^{\infty} \mathbf{K}(\vec{r}) \mathbf{A}\left(\vec{r}-\overrightarrow{r^{\prime}}\right) d \vec{r}
$$

where $\mathbf{K}$ is constructed based on intrinsic spherical simmetry for the kernel and the cumulative activity $\mathbf{A}$ is the integral over all contributions of energy emissions from point sources located at $\overrightarrow{r^{\prime}}$ positions.

Dosimetry calculation for radioisotopes is straightforwardly performed using DPK introducing the corresponding spectrum $\left(I(E)=\sum_{h} q_{h} E_{h}\right)$ 
A is the integral over all contributions of energy emissions from point sources located at $\overrightarrow{r^{\prime}}$ positions.

Dosimetry calculation for radioisotopes is straightforwardly performed using DPK introducing the corresponding spectrum $\left(I(E)=\sum_{h} q_{h} E_{h}\right)$

$$
\begin{array}{r}
D(i, j, k)=\mathbb{F}^{-1}\left\{\mathbb{F}\left\{\sum_{h} q_{h} K_{h}(i, j, k)\right\} \cdot \mathbb{F}\{A(i, j, k)\}\right\}= \\
\sum_{h} q_{h} \mathbb{F}^{-1}\left\{\mathbb{F}\left\{K_{h}(i, j, k)\right\} \cdot \mathbb{F}\{A(i, j, k)\}\right\}
\end{array}
$$

where $\mathbb{F}$ and $\mathbb{F}^{-1}$ are the fast Fourier direct and inverse transforms; respectively.

Finally, the system is capable of assessing radiobiological quantities as tumor control probability $(T C P)$, once dose distribution are calculated. Linear-quadratic or linear-quadratic-cubic models are available for the estimation of $T C P$ from absorbed dose distribution. It allows to interactively introduce radiobiological parameters associated with survival fraction and bulogical isoeffects.

\section{RESULTS AND DISCUSSIONS}

In $E D K$ and $D P K$ calculations source emission is uniformly distributed over concentric spherical regions thereby being suitable to assess energy deposition by means of a shell tally.

It was introduced the suitable adimensional quatity $X \equiv r / R_{c s d a}$ for scaling particle range in the continous slowing down approximation $\left(R_{c s d a}\right)$ in order to calculated scaled Dose Point Kernels $(s D P K)$.

The obtained $s D P K$ reported in Figure 1 were obtained performing a full stochastic approach adapting a dedicated subroutine based on FLUKA main code [4] [5].
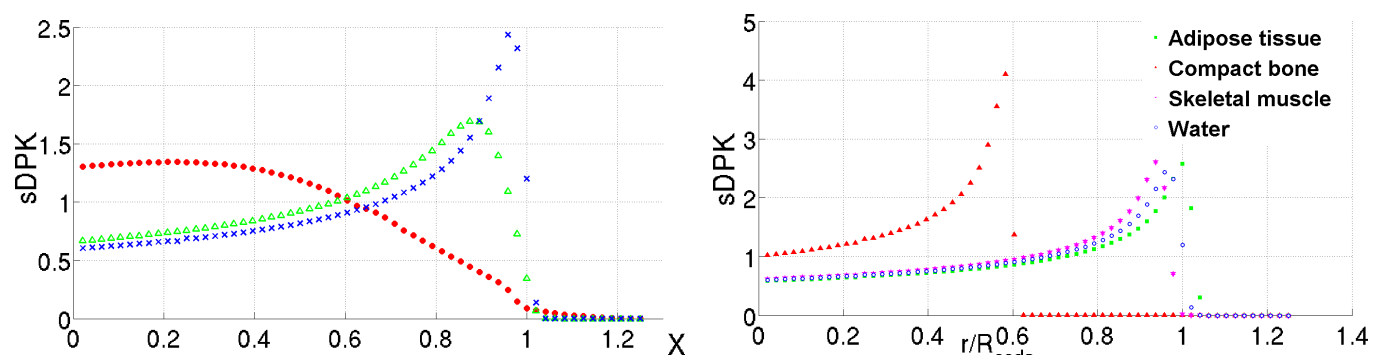

Figure 1: Left: $s D P K$ for 1 (red), 5 (green) and 10 (blue) $\mathrm{MeV}$ of $\alpha$ particles in water. Right: $s D P K$ of typical biological tissues for $10 \mathrm{MeV} \alpha$ particles.

The developed theoretical approach can be actually applied to assess internal dosimetry for any kind of therapeutic/imaging modality. As application example, a standard set of patient-specific images, both anatomic (CT) and metabolic (SPECT) have been used to test the performance of the developed model. Figure 2 shows some of the results for internal dosimetry that can be obtained with the calculation system.

Moreover, according to the purpose of the developed model, reliable 2D and 3D absorbed dose distribution should be attainable with the developed calculation system. Figures 3 and 4 present some of the available multidimensional graphics modalities of the visualization package. 

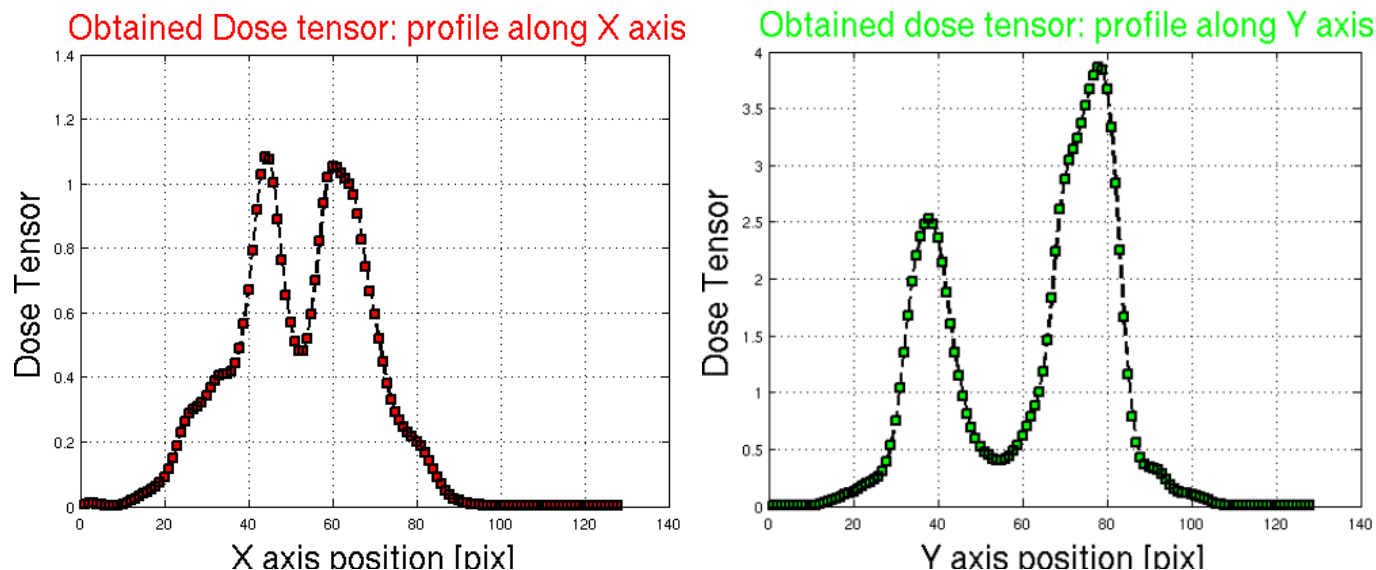

Figure 2: Dose profiles along central axes calculated by Kernel convolution approach.
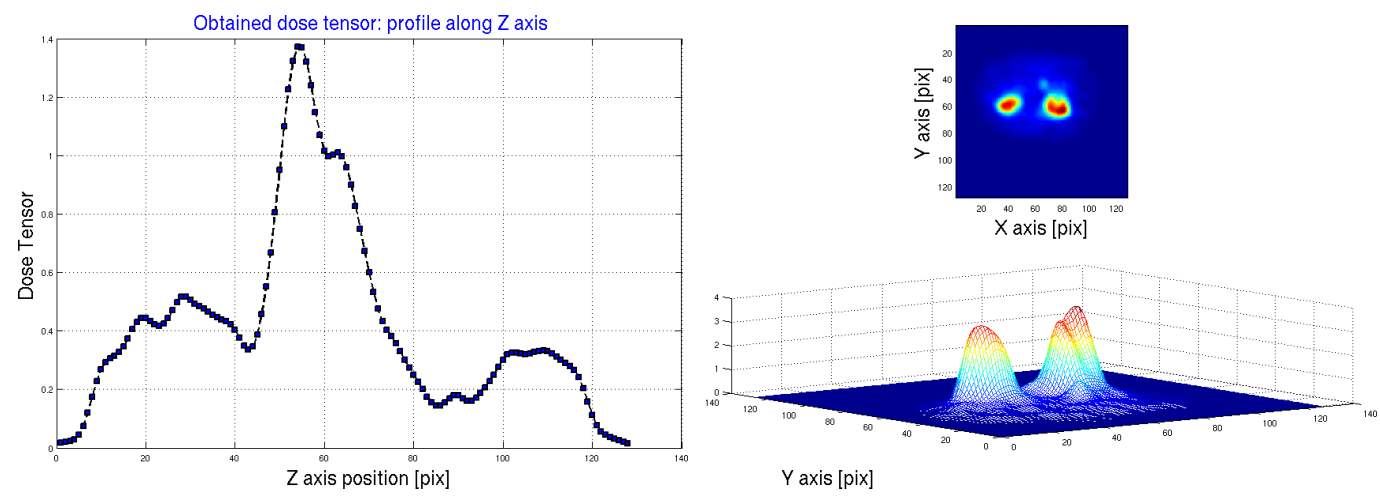

Figure 3: Dose distribution in $\mathrm{Z}$ axis (left) and XY plane (right) calculated by Kernel convolution approach.
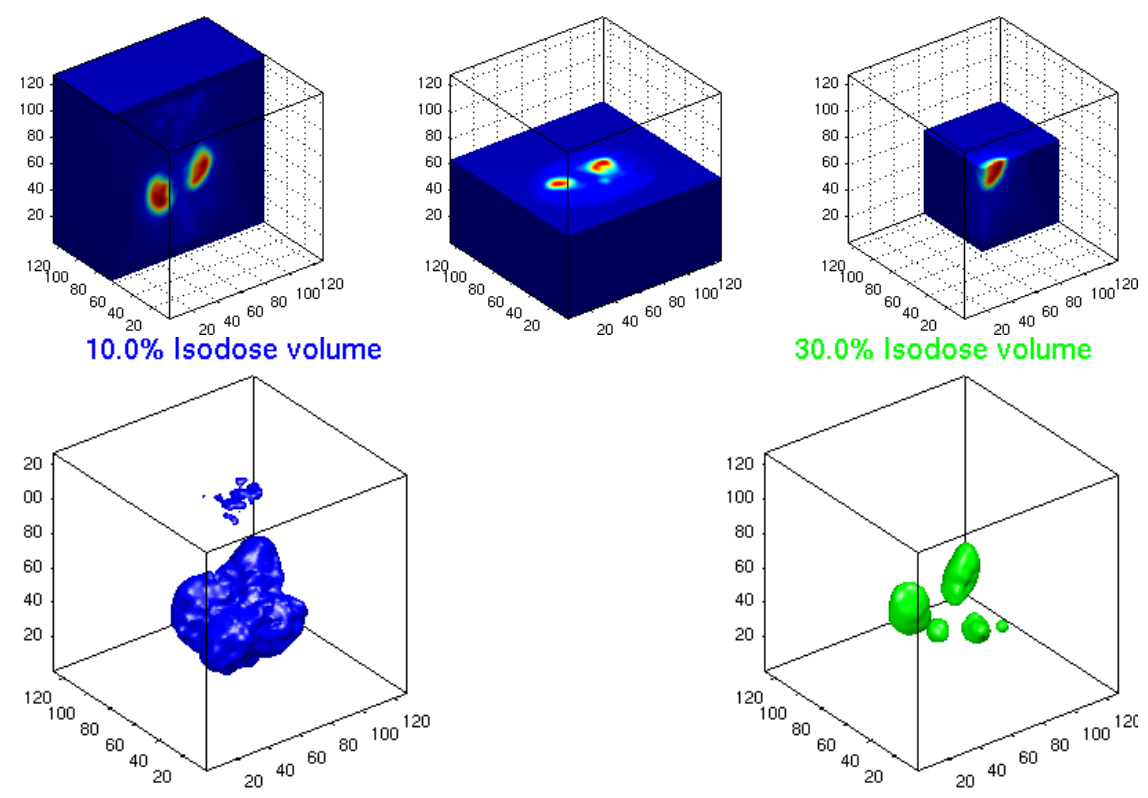

Figure 4: 3D dose distribution: 3D view with internal cuts (top) and volumes of isodose (bottom). 


\section{CONCLUSIONS}

The theory developed proposing a novel analytical-numerical approach has shown to be robust and capable of successfully performing absorbed dose distribution, even at voxel-level, for internal dosimetry as a general purpose in nuclear medicine and for $\alpha$ emitters radiopharmaceuticals internal dosimetry in particular.

The $S D P K$ were satisfactory obtained from the FLUKA main code in order to construct the necessary $\alpha$ particle $s D P K$ database to be use in the developed system.

According to preliminary benchmarking [6], the kernel convolution model has shown to be a reliable and valuable tool for fast and high performance computation of internal dosimetry aimed to nuclear medicine applications.

\section{Acknowledgments}

Authors are very grateful to FLUKA COLLABORATION and Dr. G. Battistoni, Dr. S. Muraro for their invaluable help. This work was partially supported by ISIDORA I of SeCyT-UNC and PIP 11420090100398 of CONICET projects; Argentina.

\section{References}

[1] M. Stabin. Fundamentals of nuclear medicine dosimetry. Ed. Springer, 2008.

[2] Saha G. Physics and radiobiology of nuclear medicine. Springer Inc. third edition, 2006.

[3] M. Flower and V. McCready. Radionuclide therapy dose calculations: what accuracy can be achieved?. European journal of nuclear medicine 24 (12) 1462-4, 1997.

[4] A. Ferrari, P.R. Sala, A. Fasso', and J. Ranft The FLUKA code: Description and benchmarking. CERN-2005-10, INFN/TC_05/11, SLAC-R-773, (2005)

[5] G. Battistoni, S. Muraro, P.R. Sala, F. Cerutti, A. Ferrari, S. Roesler, A. Fasso', J. Ranft. FLUKA: a multi-particle transport code. Proceedings of the Hadronic Shower Simulation Workshop 2006, Fermilab 6-8 September 2006, M.Albrow, R. Raja eds., AIP Conference Proceeding 896, 31-49 (2007)

[6] Botta F, Mairani A, Battistoni G, Cremonesi M, Di Dia A, Fassò A, Ferrari A, Ferrari M, Paganelli G, Pedroli G, Valente M. Calculation of electron and isotopes dose point kernels with FLUKA Monte Carlo code for dosimetry in nuclear medicine therapy Med. Phys. 38 (7) pp. 3944-3954 (2011) 